\title{
Phylogenetic relatedness and diversity of non- typable Haemophilus influenzae in the nasopharynx and middle ear fluid of children with acute otitis media
}

\author{
Ravinder Kaur, ${ }^{1}$ Arthur Chang, ${ }^{1}$ Qingfu Xu, ${ }^{1}$ Janet R. Casey ${ }^{2}$ \\ and Michael E. Pichichero ${ }^{1}$ \\ ${ }^{1}$ Rochester General Hospital, Research Institute, Center for Infectious Diseases and Immunology, \\ Rochester, NY, USA \\ ${ }^{2}$ Legacy Pediatrics, Rochester, NY, USA
}

Correspondence
Michael E. Pichichero

Michael.Pichichero@

rochestergeneral.org

\begin{abstract}
The phylogenetic relationships of non-typable Haemophilus influenzae (NTHi) strains prospectively isolated from healthy children and children with acute otitis media (AOM) were analysed using multilocus sequence typing (MLST). A total of $165 \mathrm{NTHi}$ isolates were collected over a 3.5 year time frame during 2006 through 2009. The strains were tested for $\beta$-lactamase production; $28.5 \%$ were positive. Seventy different NTHi sequence types (STs) were identified of which $29(41.4 \%)$ were novel. NTHi strains did not show any phylogenetic grouping or clustering among asymptomatic colonizing strains or strains that caused AOM, or based on $\beta$-lactamase enzyme production. Evaluation of triplets and other siblings over time demonstrated relatively frequent genetic exchanges in NTHi isolates in vivo in a short time frame and subsequent transfer among children in a family. Comparison of the MLST STs isolated at different time points showed that in $\sim 85 \%$ of the nasopharynx (NP) colonizations, NTHi strains cleared from the host within 3 months, that sequential colonization in the same child involved different strains in all cases except one, and that NP and middle ear isolates were identical STs in $84 \%$ of cases. In this first study of its type to our knowledge, we could not identify predominant MLST types among strains colonizing the NP versus those causing AOM or expressing a $\beta$-lactamase enzyme conferring penicillin resistance in children.
\end{abstract}

Received 4 May 2011

Accepted 22 July 2011

\section{INTRODUCTION}

Non-typable Haemophilus influenzae (NTHi) is an important human-specific pathogen colonizing the nasopharynx (NP) of the upper respiratory tract (Faden et al., 1995; Murphy et al., 2009). NTHi strains are rarely associated with invasive disease in children, but they are associated with respiratory tract infections in both children and adults (Murphy, 2003; Kyd \& Cripps, 1999). NP colonization with NTHi is an important risk factor for developing disease (Faden et al., 1997). Children frequently NP-colonized by NTHi are more likely to develop acute otitis media (AOM) than less frequently colonized children (Faden et al., 1997; Harabuchi et al., 1994). Simultaneous detection of multiple isolates of NTHi from the same sample has been reported among aboriginal children with otitis media (Smith-Vaughan et al., 1996), among otitis-prone children in day care centres (St Sauver et al., 2000) and in cystic fibrosis patients (Bandi et al.,

Abbreviations: AOM, acute otitis media; MEF, middle ear fluid; MLST, multilocus sequence typing; NP, nasopharynx; NTHi, non-typable Haemophilus influenzae; ST, sequence type; TS, throat swab.
2001; Murphy et al., 1999), which may result from inadequate clearance of colonizing bacteria, exposure to multiple circulating strains or in vivo genetic recombinations in a specific host. It is not known whether NTHi isolates that express $\beta$-lactamases (responsible for resistance to $\beta$-lactam antibiotics such as penicillins, cephamycins and carbapenems) are phylogenetically related. Various studies of Streptococcus pneumoniae have shown phylogenetic relationships among antibiotic-resistant strains (Beall et al., 2006; Hanage et al., 2009; Porat et al., 2004; Xu et al., 2009). In this study, we explored whether or not NTHi shows genetic relatedness in relation to $\beta$-lactamase enzyme production. It is also not known whether NTHi strains that cause disease such as AOM are genetically clustered and/or distinct from isolates associated with asymptomatic NP colonization.

Using outer-membrane profiles, Faden et al. (1995) reported rapid disappearance of initial colonizing strains of $H$. influenzae. An immune response was shown to eliminate a particular strain and prevent its reacquisition but not the acquisition of heterologous strains. Interestingly, prolonged colonization with the same strain in children was also 
observed (Faden et al., 1995). Similar observations were made regarding AOM; that is, immune responses to homologous strains of NTHi prevented subsequent AOM from the same strain but not heterologous strains (Barenkamp et al., 1984).

To study the immune response following natural exposure to NTHi during NP colonization and AOM in conjunction with molecular characterization, by multilocus sequence typing (MLST), of strains would be highly discriminatory. To our knowledge, this is the first study aimed at exploring phylogenetic relationships among NTHi strains based on MLST in prospectively collected samples from young children.

\section{METHODS}

Patient population, sampling and bacterial isolation. Samples of NTHi were collected from children participating in a National Institute of Deafness and Communication Disorders-supported study on NTHi pathogenesis and immunity. The children were derived from a suburban, private paediatric practice (Legacy Pediatrics) in Rochester, New York, USA. For this report, samples were collected for the 3.5 year time span of June 2006-December 2009. Children were enrolled at 6 months of age and then sequentially sampled at 9, 12, 15, 18,24 and 30 months of age in their routine healthy visits. At each visit, an NP sample and a throat swab (TS) were obtained. Whenever a child became ill with AOM, middle ear fluid (MEF) was collected by tympanocentesis and NP and TS samples were obtained. Then, 3-4 weeks after an AOM visit, NP and TS follow-up samples were obtained. In addition, otitis-prone children who had $\geqslant$ three episodes of AOM in 6 months or $\geqslant$ four episodes in a year were enrolled but not routinely followed longitudinally and MEF and NP samples were obtained from these children. NTHi bacteria were isolated and identified according to standard laboratory procedures including colony morphology, porphyrin reactivity, and growth requirement for haemin and nicotinamide adenine dinucleotide and growth on Haemophilus ID Quad plates. Only one colony of NTHi from each sample was cultured and stored at $-80{ }^{\circ} \mathrm{C}$ for further experiments. An isolate was further identified as NTHi as described by Murphy et al. (2007), including ompP6 sequencing to distinguish NTHi from Haemophilus haemolyticus. $\beta$-Lactamase testing was performed on all NTHi isolates by Cefinase disc test.

MLST. Bacterial genomic DNA was extracted from pure cultures of NTHi isolated from NP, TS or MEF samples (if children had AOM). The internal fragments of seven housekeeping genes of NTHi were amplified by PCR, using a PCR Master Mix (Promega) as described previously (Meats et al., 2003). The PCR products were purified using the ExoSAP kit (USB) and identified by DNA sequencing. The sequences of seven genes for each isolate were submitted to the international MLST database (http://haemophilus.mlst.net) to obtain the sequence type (ST). Two strains are defined as a clone if they are single-locus variants of seven housekeeping genes.

\section{RESULTS}

A total of 168 children were enrolled and followed longitudinally from June 2006 to December 2009. NTHi was detected in $73(43.5 \%)$ children at least once, and among these 73 children, NTHi colonization was observed more than once in 33 (45.2\%) children.

A total of 165 NTHi isolates were characterized by MLST. We could not obtain a sequence for all seven genes for six of the NTHi isolates and therefore the ST was not found. We found 70 different MLST STs among the NTHi isolates; 29 were novel and submitted to the $H$. influenzae international database. Phylogenetic analysis was conducted on different strains with MEGA4 software (Tamura et al., 2007). The evolutionary history was inferred using the neighbourjoining method. The evolutionary distances were computed using the maximum composite likelihood method and are in units of the number of base substitutions per site. Fig. 1 shows the genetic relatedness of NTHi strains circulating in children of the Rochester community. Strains were grouped based on those that were associated with NP/TS colonization versus strains that caused AOM. No pattern/grouping was found. The relatedness of $\beta$-lactamase-positive $(+)$ and $\beta$-lactamasenegative (-) strains is also shown in Fig. 1. Overall, NTHi STs did not show clustering based on $\beta$-lactamase testing. A very small cluster of $\beta$-lactamase-positive strains where MLST STs were in the same clonal complex (i.e. ST103, 145 614, 701) were observed in the phylogenetic tree (Fig. 1). With additional NTHi strains and their $\beta$-lactamase susceptibility information, phylogenetic clustering may be clearer in the future.

We also used the eBURST method (http://haemophilus.mlst. net/eburst) as a way of displaying the relationships between closely related isolates of a bacterial species or population to define groups. The eBURST, unlike cluster diagrams, trees or dendrograms, uses a simple but appropriate model of bacterial evolution in which an ancestral (or founding) genotype increases in frequency in the population, and while doing so, begins to diversify to produce a cluster of closely related genotypes that are all descended from the founding genotype. This cluster of related genotypes is referred to as a 'clonal complex'. In eBURST analysis, we defined groups in which each NTHi ST was identical at five or more of the seven sequenced loci to at least one other NTHi ST in the group. The NTHi isolates from our study population were present in 19 of these 29 eBURST groups of all NTHi found in the $H$. influenzae MLST website. Table 1 summarizes the eBURST group analysis to show where the NTHi isolates belong. Twenty-nine such groups contained five or more STs. In our study, $27.9 \%$ of the total 165 NTHi isolates or $18.7 \%$ of the total 70 NTHi STs belonged to eBURST group 2 . The remainder of the isolates were in different eBURST groups. These results suggest that the NTHi population was dispersed in several distinct genetic groups.

\section{Comparison of NTHi strains isolated at different visits}

Fig. 2 shows the comparison of NTHi according to STs isolated at different time points in 33 children. In most cases, the NTHi strains cleared from the child within the 3month time interval between samplings. Sequential NTHi NP colonization observed at different time points in most children involved different strains. We did not observe any reacquisition of previously carried NTHi strains except in one case (child 06-02-045). For this one child, NTHi ST575 


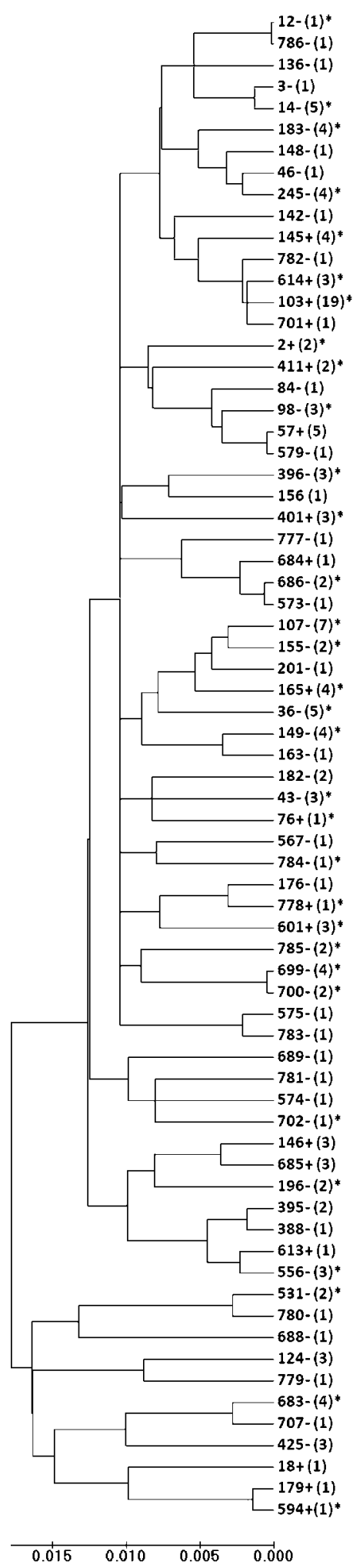

was isolated at the 6-month visit. At the 9-month visit, a different NTHi strain was isolated. At the next visit at 12 months of age, ST783 was observed. Since ST783 is the same clone as ST575, it might be that we missed this clone at the 9-month visit.
Fig. 1. Phylogenetic tree from concatenated sequences of MLST of $70 \mathrm{NTHi}$ strains isolated from Rochester NY children. The linkage distance is shown at the bottom of the dendrogram and is in the units of the number of base substitutions per site. The number of isolates cultured for each ST is shown in parentheses. The strains that were found in MEF of children with AOM are indicated by an asterisk. $\beta$-Lactamase results are also shown for each strain with $\mathrm{a}+$ and - symbol after the strain number.

ST103 was the most common strain found. In $24.2 \%$ of the 33 children where NTHi colonization was observed more than once, prolonged colonization was observed with the same ST. STs 146, 182 and 685 remained in the NP of children asymptomatically for at least 3, 3 and 6 months, respectively. ST699 persisted in a child for 9 months and remained colonizing the NP during two episodes of AOM and was still present in the NP after two courses of antibiotic. Similarly, ST149 persisted in a child for 6 months and caused two episodes of AOM and did not clear the NP after either antibiotic course. In $\sim 36 \%$ of the AOM cases, NTHi did not clear from the NP of the child after antibiotic treatment.

\section{NTHi strain comparison in MEF versus NP}

The comparison of NTHi isolates cultured from MEF versus NP/TS samples from 37 children with AOM is also shown in Table 2. In $84 \%$ of the children, the same ST was found in the MEF as in the NP/TS sample. In $8 \%$ of the cases, a different ST, but the same clone, was found in the $\mathrm{NP}$ versus MEF. In $8 \%$ of the children, a different clone was detected in the MEF compared to the NP/TS, suggesting that two strains of NTHi were colonizing the $\mathrm{NP}$ and either we did not isolate the strain from the NP sample that was successful in ascending from the NP to the middle ear to cause AOM, or the major colonizer was not causing disease.

\section{NTHi carriage in triplets}

The comparison of MLST profiles of NTHi strains isolated at different points from a family of triplets who had an older sibling is shown in Table 3. The MLST profiles of the strains from the triplets are consistent with likely genetic exchange of NTHi in the NP of a child in a short time frame followed by dissemination of the new strain to siblings in the family. Triplet 1 carried ST103 and triplets 2 and 3 carried the same clone, ST614, around the same time. Similarly, triplet 2 carried ST556 and triplet 1 carried ST613, which is the same clone.

\section{DISCUSSION}

The identification of clearly defined groups of strains in a genotype is a central problem in microbiology because of very frequent localized homologous recombination in 
Table 1. eBURST group analysis of the $H$. influenzae database showing where the NTHi STs from this study belong

The first column shows the eBURST group and the last column shows the ST found in that group in our study. The number of different STs and number of isolates found in each group are also shown. Percentages in parentheses are from the total number of 70 STs and total number of 165 NTHi isolates from this study population.

\begin{tabular}{|c|c|c|c|}
\hline $\begin{array}{l}\text { eBURST group from } H \text {. } \\
\text { influenzae database }\end{array}$ & $\begin{array}{l}\text { No. of different STs present } \\
\text { in the eBURST group (\%) }\end{array}$ & $\begin{array}{l}\text { No. of isolates present in } \\
\text { the eBURST group (\%) }\end{array}$ & $\begin{array}{c}\text { ST number of NTHi isolates from chidren } \\
\text { during NP colonization and AOM }\end{array}$ \\
\hline Group 2 & $13(18.5)$ & $46(27.9)$ & $\begin{array}{l}136,3,14,183,148,46,245,145,782,614,103 \\
701,777\end{array}$ \\
\hline Group 3 & $6(8.6)$ & $9(5.5)$ & $684,686,573,176,778,601$ \\
\hline Group 4 & $1(1.4)$ & $1(0.6)$ & 18 \\
\hline Group 5 & $1(1.4)$ & $3(1.8)$ & 124 \\
\hline Group 8 & $2(2.9)$ & $5(3.0)$ & 149,163 \\
\hline Group 9 & $2(2.9)$ & $6(3.6)$ & 396,401 \\
\hline Group 10 & $1(1.4)$ & $2(1.2)$ & 411 \\
\hline Group 12 & $2(2.9)$ & $5(3.0)$ & 683,707 \\
\hline Group 13 & $1(1.4)$ & $1(0.6)$ & 84 \\
\hline Group 15 & $1(1.4)$ & $1(0.6)$ & 574 \\
\hline Group 21 & $2(2.9)$ & $2(1.2)$ & 12,786 \\
\hline Group 22 & $1(1.4)$ & $3(1.8)$ & 425 \\
\hline Group 23 & $1(1.4)$ & $1(0.6)$ & 156 \\
\hline Group 26 & $1(1.4)$ & $2(1.2)$ & 785 \\
\hline Groups contain less than 5 STs & $14(20)$ & $29(17.6)$ & $\begin{array}{l}142,165,182,43,567,699,700,575,783,146 \\
685,196,179,594\end{array}$ \\
\hline Singletons & $8(11.4)$ & $13(7.9)$ & $2,36,76,689,781,702,688,779$ \\
\hline
\end{tabular}

bacteria (Stackebrandt et al., 2002). In this paper, we have examined one of the more highly recombinogenic species, $H$. influenzae, to explore the extent to which this species can be resolved into distinct populations using multilocus sequencing types. We addressed the question of whether NTHi strains that asymptomatically colonize the NP are phylogenetically distinct from NTHi strains that cause AOM, and found they were not. We also explored the possibility that strains expressing a $\beta$-lactamase enzyme (which confers penicillin resistance) might cluster phylogenetically, and found they did not. Previously, a study by Meats et al. (2003) has shown phylogenetic diversity between all the strains of $H$. influenzae using the MLST approach. Our results are consistent with the observations of Erwin et al. (2008), who showed considerable diversity within NTHi strains. Erwin et al. (2008) found 13 clades or 10 eBURST groups from the $H$. influenzae MLST database. Eighty per cent of their NTHi strains were in group 2. With more strains added to the database, we found new eBURST groups (29 eBURST groups compared to 10 groups in their study using the same group definition). Specifically, $41.4 \%$ of the NTHi STs found in our study were distributed in 10 eBURST groups from the Erwin et al. (2008) study but $27.1 \%$ of the STs from our study were found in new eBURST groups, and $31.5 \%$ of the NTHi STs we evaluated did not correspond to any of the 29 eBURST groups.
We found that asymptomatic colonizing strains were not phylogenetically distinct from strains that caused AOM disease. The NTHi strain that only caused colonization in one child was responsible for AOM disease in a different child. We interpret this result to suggest that either the progression from asymptomatic colonization to AOM may not be dependent on the strain of NTHi but rather on quantitative or qualitative differences in the immune defence of the child, or other factors such as differential expression of specific adhesins might be also involved, apart from difference in immune defence.

We could not find any previous studies on clustering of $H$. influenzae MLST types based on antibiotic resistance. With more extensive characterization of isolates of Streptococcus pneumoniae, another common respiratory bacterium, clustering based on antibiotic resistance has been observed (Hanage et al., 2009; Xu et al., 2009). A very small cluster of $\beta$-lactamase-positive strains where MLST STs were in the same clonal complex was seen in the phylogenetic tree in our study of 70 different STs. As a larger database of $H$. influenzae strains evolves, clusters of NTHi that are antibiotic resistant may emerge. Since $\beta$-lactamase is generally carried by a plasmid, it would not be surprising if different STs producing $\beta$-lactamase or not are present in the same clonal complex. We also found that the same 


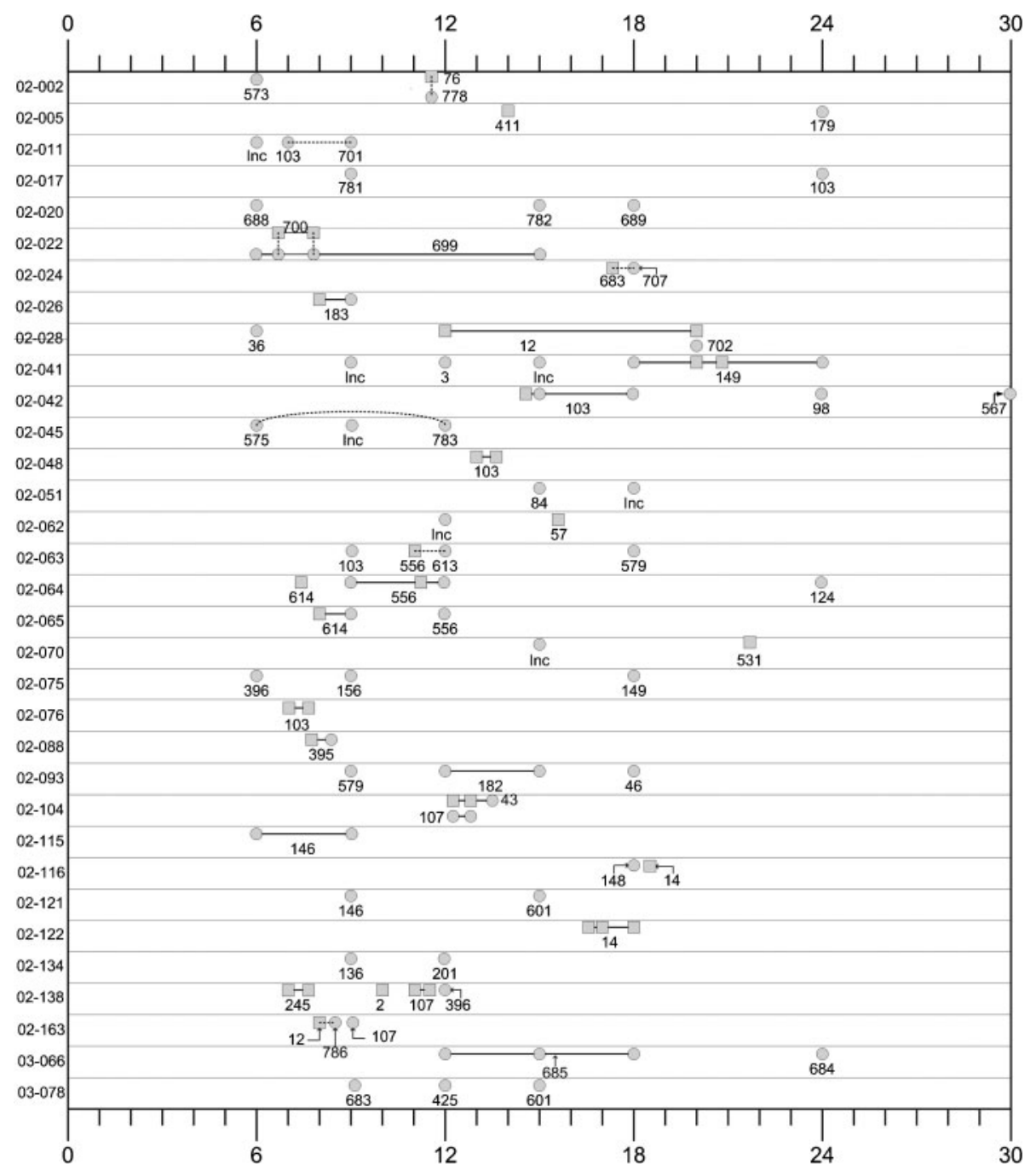

Fig. 2. Comparison of MLST STs obtained from NTHi isolates at different time points from the same subjects. The $x$-axis represents the child's age in months and the $y$-axis indicates the child's identification number. Circles represent NTHi strains colonizing the NP; squares represent NTHi strains found in MEF. Two isolates of NTHi connected by a line are the same ST, and those connected by a dashed line are different STs, but the same clone (single-locus variant). Inc means it was not possible to obtain a sequence for all seven genes for that isolate and therefore the ST was not obtained, but partial analysis clearly allowed us to determine that the strain was different from the other strains in that particular child.

clone with a different ST may or may not have $\beta$-lactamase activity (e.g. ST776 and ST176). Others have also found different antibiotic susceptibility among strains of Staphylococcus aureus (Enright et al., 2000) and Streptococcus pneumoniae (Feil et al., 2000) in the same clonal complex. A study by Hotomi et al. (2007) has demonstrated clonal dissemination of $\beta$-lactamase non-producing ampicillinresistant (BLNAR) $H$. influenzae strains isolated from the upper respiratory tract in Japan but the BLNAR strains carried modifications in their pencillin-binding proteins, encoded by the ftsI gene that is present in the chromosome.

We found a frequent turnover of NTHi strains among children. When sequential NTHi NP colonization was observed at different time points in a child, different STs were involved. Faden et al. (1995) found that NTHi strains cleared the NP in $<3$ months in $90 \%$ of the children they studied. We did not observe any reacquisition of previously carried NTHi strains, similar to previous studies by Spinola et al. (1986) and Faden et al. (1995).

Prolonged colonization of NTHi strains ( $>3$ months) was observed in $\sim 25 \%$ of the 33 children where NTHi colonization was observed more than once. ST146 and ST685 belong to the same clonal complex and have no predictor founder. Based on the information in the $H$. influenzae MLST database, ST146 was first reported from Michigan in 1998 as an NP carriage strain but later was isolated from blood in cases of bacteraemia in California (2000) and Colorado (2002). There are no exhaustive studies on NTHi 
Table 2. Comparison of MLST STs of NTHi isolates cultured from different sites (NP, TS and left and right MEF) from children with $\mathrm{AOM}$ at the same visit to determine whether the isolates were of the same or a different clone

\begin{tabular}{|c|c|c|c|c|c|c|c|c|c|c|}
\hline \multirow[t]{2}{*}{ Subject } & \multirow[t]{2}{*}{ Source } & \multirow[t]{2}{*}{ ST } & \multicolumn{7}{|c|}{ Allele number of housekeeping gene } & \multirow[t]{2}{*}{ Comparison } \\
\hline & & & $a d k$ & $\operatorname{atpG}$ & $f r d B$ & fucK & $m d h$ & $p g i$ & $\operatorname{rec} A$ & \\
\hline $06-02-003$ & NP/LMEF & 57 & 14 & 7 & 13 & 7 & 17 & 13 & 17 & Same \\
\hline 06-01-013 & NP/LMEF & 396 & 10 & 2 & 15 & 8 & 26 & 61 & 3 & Same \\
\hline 06-01-022 & NP/LMEF & 196 & 14 & 8 & 18 & 11 & 17 & 2 & 3 & Same \\
\hline 06-01-023 & TS/LMEF & 145 & 1 & 8 & 1 & 14 & 22 & 14 & 13 & Same \\
\hline 06-01-025 & TS/RMEF & 145 & 1 & 8 & 1 & 14 & 22 & 14 & 13 & Same \\
\hline 06-01-009 & NP/RMEF & 103 & 1 & 1 & 1 & 14 & 9 & 14 & 13 & Same \\
\hline 06-01-039 & NP/LMEF & 686 & 7 & 3 & 42 & 1 & 66 & 1 & 29 & Same \\
\hline $06-01-043$ & TS/LMEF & 98 & 14 & 7 & 13 & 15 & 17 & 13 & 1 & Same \\
\hline $06-01-048$ & NP/LMEF & 165 & 44 & 2 & 16 & 37 & 17 & 2 & 3 & Same \\
\hline 06-01-054 & NP/LMEF & 36 & 16 & 21 & 14 & 16 & 29 & 14 & 3 & Same \\
\hline 06-01-056 & TS/RMEF & 401 & 11 & 2 & 15 & 8 & 28 & 26 & 21 & Same \\
\hline 07-01-060 & NP/LMEF & 395 & 14 & 51 & 16 & 48 & 15 & 2 & 31 & Same \\
\hline 07-01-061 & NP/RMEF & 36 & 16 & 21 & 14 & 16 & 29 & 14 & 3 & Same \\
\hline \multirow[t]{2}{*}{$07-02-064$} & NP & 614 & 1 & 1 & 1 & 8 & 9 & 14 & 13 & Different ST but same clone \\
\hline & RMEF & 103 & 1 & 1 & 1 & 14 & 9 & 14 & 13 & \\
\hline \multirow[t]{2}{*}{$06-02-022$} & NP & 699 & 114 & 5 & 38 & 73 & 15 & 158 & 106 & Different ST but same clone \\
\hline & RMEF/LMEF & 700 & 114 & 1 & 38 & 73 & 15 & 158 & 106 & \\
\hline $06-02-024$ & NP/RMEF & 683 & 93 & 41 & 41 & 8 & 69 & 60 & 98 & Same \\
\hline 06-01-011 & NP/LMEF & 57 & 14 & 7 & 13 & 7 & 17 & 13 & 17 & Same \\
\hline 06-01-020 & TS/LMEF & 411 & 14 & 7 & 13 & 30 & 1 & 21 & 1 & Same \\
\hline 06-01-031 & NP/RMEF & 165 & 44 & 2 & 16 & 37 & 17 & 2 & 3 & Same \\
\hline \multirow[t]{2}{*}{ 08-03-075 } & RMEF & 601 & 5 & 33 & 7 & 1 & 112 & 41 & 29 & Different clone \\
\hline & NP & 149 & 3 & 9 & 8 & 13 & 14 & 8 & 4 & \\
\hline \multirow[t]{2}{*}{$06-02-002$} & RMEF & 778 & 5 & 33 & 7 & 15 & 47 & 58 & 15 & Different ST but same clone \\
\hline & NP & 176 & 5 & 33 & 7 & 15 & 47 & 58 & 29 & \\
\hline $06-02-141$ & NP/RMEF & 149 & 3 & 9 & 8 & 13 & 14 & 8 & 4 & Same \\
\hline 06-02-048 & NP/RMEF/LMEF & 103 & 1 & 1 & 1 & 14 & 9 & 14 & 13 & Same \\
\hline $07-02-062$ & NP/LMEF & 57 & 14 & 7 & 13 & 7 & 17 & 13 & 17 & Same \\
\hline 07-02-064 & NP/LMEF & 556 & 14 & 51 & 16 & 48 & 29 & 2 & 31 & Same \\
\hline $08-02-076$ & NP/RMEF/LMEF & 103 & 1 & 1 & 1 & 14 & 9 & 14 & 13 & Same \\
\hline 08-02-088 & NP/LMEF & 395 & 14 & 51 & 16 & 48 & 15 & 2 & 31 & Same \\
\hline \multirow[t]{2}{*}{ 08-02-104 } & NP & 107 & 33 & 8 & 16 & 16 & 49 & 2 & 3 & Different clone \\
\hline & RMEF & 43 & 1 & 22 & 19 & 19 & 32 & 17 & 2 & \\
\hline 09-02-122 & NP/LMEF & 14 & 5 & 1 & 1 & 1 & 1 & 2 & 5 & Same \\
\hline 09-02-130 & NP/LMEF & 785 & 55 & 11 & 16 & 76 & 89 & 113 & 3 & Same \\
\hline 09-02-132 & NP/LMEF/RMEF & 156 & 26 & 2 & 15 & 7 & 22 & 56 & 3 & Same \\
\hline \multirow[t]{2}{*}{ 09-02-136 } & NP & 396 & 10 & 2 & 15 & 8 & 26 & 61 & 3 & Different clone \\
\hline & MEF & 107 & 33 & 8 & 16 & 16 & 49 & 2 & 3 & \\
\hline 09-02-138 & NP/LMEF/RMEF & 245 & 1 & 24 & 18 & 18 & 27 & 1 & 5 & Same \\
\hline 09-02-138 & NP/LMEF & 107 & 33 & 8 & 16 & 16 & 49 & 2 & 3 & Same \\
\hline 09-02-138 & NP/LMEF & 2 & 14 & 7 & 1 & 15 & 16 & 4 & 1 & Same \\
\hline 09-02-161 & NP/LMEF & 165 & 44 & 2 & 16 & 37 & 17 & 2 & 3 & Same \\
\hline 09-02-163 & NP/LMEF & 12 & 1 & 1 & 1 & 13 & 13 & 25 & 16 & Same \\
\hline
\end{tabular}

MLST types in invasive disease, so we cannot determine whether ST146 and ST685 are associated or not with invasive disease.

In $\sim 36 \%$ of our subjects, NTHi strains persisted in the NP or middle ear after antibiotic treatment, a finding similar to that of van Schilfgaarde et al. (1999) and others (Cohen et al., 1999; Dabernat et al., 1998). Strains that persist in the $\mathrm{NP}$ after antibiotic therapy may be the result of antibiotic resistance or other unique characteristics of the strain or could be a consequence of lower levels of antibiotic in a host due to suboptimal absorption of the drug (Pichichero et al., 2008; Pichichero \& Reed, 2009).

We found that in most of the children in our study, NTHi strains present in the MEF were identical to the strains isolated from the NP, consistent with previous studies (Murphy et al., 1987; Barenkamp et al., 1984). 
Table 3. Comparison of NTHi MLST STs obtained from one family that included triplets and one adopted sibling

Results are shown from different visits demonstrating the transmission of NTHi isolates. ND, Not possible to obtain a sequence for this locus from this isolate and therefore the ST was not obtained, indicated by a - sign.

\begin{tabular}{|c|c|c|c|c|c|c|c|c|c|c|}
\hline \multirow[t]{2}{*}{ Patient no. } & \multirow[t]{2}{*}{ Date of visit } & \multirow[t]{2}{*}{ Age (months) } & \multicolumn{7}{|c|}{ Allele number of housekeeping gene } & \multirow[t]{2}{*}{ ST } \\
\hline & & & $a d k$ & $\operatorname{atp} G$ & $f r d B$ & fucK & $m d h$ & $p g i$ & $\operatorname{rec} A$ & \\
\hline \multirow{2}{*}{ 07-02-063 Triplet 1} & $13-2-2008$ & 9.5 & 1 & 1 & 1 & 14 & 9 & 14 & 13 & 103 \\
\hline & $13-5-2008$ & 12.5 & 14 & 5 & 16 & 48 & 29 & 2 & 31 & 613 \\
\hline 07-02-064 Triplet 2 & $18-1-2008$ & 8.5 & 1 & 1 & 1 & 8 & 9 & 14 & 13 & 614 \\
\hline \multirow[t]{3}{*}{ 07-02-065 Triplet 3} & $29-1-2008$ & 9 & 1 & 1 & 1 & 8 & 9 & 14 & 13 & 614 \\
\hline & $13-2-2008$ & 9.5 & 1 & 1 & 1 & 8 & 9 & 14 & 13 & 614 \\
\hline & $13-5-2008$ & 12.5 & 14 & 51 & 16 & 48 & 29 & 2 & 31 & 556 \\
\hline \multirow[t]{2}{*}{ 06-02-042 Sibling } & $7-1-2008$ & 18.5 & 1 & 1 & 1 & 14 & 9 & 14 & 13 & 103 \\
\hline & $23-1-2008$ & 19 & 1 & 1 & 1 & 14 & 9 & 14 & 13 & 103 \\
\hline
\end{tabular}

A known mechanism for new NTHi strain acquisition is by close contact among individuals (Loos et al., 1989). Family contacts would be likely to carry the same NTHi strains at any one time. Our results from examination of the MLST profiles of strains isolated from triplets and their sibling at different time points clearly demonstrate this phenomenon.

Although this is the largest study of NTHi isolates in young children, the ability to show phylogenetic relatedness is constrained by the number of isolates. Further study of phylogenetic relationships among NTHi strains would be useful. Clustering among strains that asymptomatically colonize the NP or are able to cause local infection in the middle ear or sinuses or lungs may yet emerge. Improving our understanding of the biology of NTHi may prove valuable in strategies to prevent NP colonization and progression to local respiratory infections.

\section{ACKNOWLEDGEMENTS}

This work was supported by NIH, NIDCD RO1DC008671-01A2 (to M.E.P.). The authors gratefully acknowledge Sally Thomas, LPN, CCRC, the nurses and staff of Legacy Pediatrics and the collaborating paediatricians from Sunrise Pediatrics, Westfall Pediatrics, Lewis Pediatrics and Long Pond Pediatrics, the parents who consented and the children who participated in this long and challenging study.

\section{REFERENCES}

Bandi, V., Apicella, M. A., Mason, E., Murphy, T. F., Siddiqi, A., Atmar, R. L. \& Greenberg, S. B. (2001). Nontypeable Haemophilus influenzae in the lower respiratory tract of patients with chronic bronchitis. Am J Respir Crit Care Med 164, 2114-2119.

Barenkamp, S. J., Shurin, P. A., Marchant, C. D., Karasic, R. B., Pelton, S. I., Howie, V. M. \& Granoff, D. M. (1984). Do children with recurrent Haemophilus influenzae otitis media become infected with a new organism or reacquire the original strain? J Pediatr 105, 533-537.
Beall, B., McEllistrem, M. C., Gertz, R. E., Jr, Wedel, S., Boxrud, D. J., Gonzalez, A. L., Medina, M. J., Pai, R., Thompson, T. A. \& other authors (2006). Pre- and postvaccination clonal compositions of invasive pneumococcal serotypes for isolates collected in the United States in 1999, 2001, and 2002. J Clin Microbiol 44, 999-1017.

Cohen, R., Navel, M., Grunberg, J., Boucherat, M., Geslin, P., Derriennic, M., Pichon, F. \& Goehrs, J. M. (1999). One dose ceftriaxone vs. ten days of amoxicillin/clavulanate therapy for acute otitis media: clinical efficacy and change in nasopharyngeal flora. Pediatr Infect Dis J 18, 403-409.

Dabernat, H., Geslin, P., Megraud, F., Bégué, P., Boulesteix, J., Dubreuil, C., de La Roque, F., Trinh, A. \& Scheimberg, A. (1998). Effects of cefixime or co-amoxiclav treatment on nasopharyngeal carriage of Streptococcus pneumoniae and Haemophilus influenzae in children with acute otitis media. J Antimicrob Chemother 41, 253258.

Enright, M. C., Day, N. P., Davies, C. E., Peacock, S. J. \& Spratt, B. G. (2000). Multilocus sequence typing for characterization of methicillin-resistant and methicillin-susceptible clones of Staphylococcus aureus. J Clin Microbiol 38, 1008-1015.

Erwin, A. L., Sandstedt, S. A., Bonthuis, P. J., Geelhood, J. L., Nelson, K. L., Unrath, W. C., Diggle, M. A., Theodore, M. J., Pleatman, C. R. \& other authors (2008). Analysis of genetic relatedness of Haemophilus influenzae isolates by multilocus sequence typing. J Bacteriol 190, 14731483.

Faden, H., Duffy, L., Williams, A., Krystofik, D. A. \& Wolf, J. (1995). Epidemiology of nasopharyngeal colonization with nontypeable Haemophilus influenzae in the first 2 years of life. J Infect Dis 172, 132-135.

Faden, H., Duffy, L., Wasielewski, R., Wolf, J., Krystofik, D. \& Tung, Y. (1997). Relationship between nasopharyngeal colonization and the development of otitis media in children. Tonawanda/Williamsville Pediatrics. J Infect Dis 175, 1440-1445.

Feil, E. J., Smith, J. M., Enright, M. C. \& Spratt, B. G. (2000). Estimating recombinational parameters in Streptococcus pneumoniae from multilocus sequence typing data. Genetics 154, 1439-1450.

Hanage, W. P., Fraser, C., Tang, J., Connor, T. R. \& Corander, J. (2009). Hyper-recombination, diversity, and antibiotic resistance in pneumococcus. Science 324, 1454-1457. 
Harabuchi, Y., Faden, H., Yamanaka, N., Duffy, L., Wolf, J. \& Krystofik, D. (1994). Nasopharyngeal colonization with nontypeable Haemophilus influenzae and recurrent otitis media. Tonawanda/ Williamsville Pediatrics. J Infect Dis 170, 862-866.

Hotomi, M., Fujihara, K., Billal, D. S., Suzuki, K., Nishimura, T., Baba, S. \& Yamanaka, N. (2007). Genetic characteristics and clonal dissemination of beta-lactamase-negative ampicillin-resistant Haemophilus influenzae strains isolated from the upper respiratory tract of patients in Japan. Antimicrob Agents Chemother 51, 3969-3976.

Kyd, J. \& Cripps, A. (1999). Nontypeable Haemophilus influenzae: challenges in developing a vaccine. J Biotechnol 73, 103-108.

Loos, B. G., Bernstein, J. M., Dryja, D. M., Murphy, T. F. \& Dickinson, D. P. (1989). Determination of the epidemiology and transmission of nontypable Haemophilus influenzae in children with otitis media by comparison of total genomic DNA restriction fingerprints. Infect Immun 57, 2751-2757.

Meats, E., Feil, E. J., Stringer, S., Cody, A. J., Goldstein, R., Kroll, J. S., Popovic, T. \& Spratt, B. G. (2003). Characterization of encapsulated and noncapsulated Haemophilus influenzae and determination of phylogenetic relationships by multilocus sequence typing. J Clin Microbiol 41, 1623-1636.

Murphy, T. F. (2003). Respiratory infections caused by non-typeable Haemophilus influenzae. Curr Opin Infect Dis 16, 129-134.

Murphy, T. F., Bernstein, J. M., Dryja, D. M., Campagnari, A. A. \& Apicella, M. A. (1987). Outer membrane protein and lipooligosaccharide analysis of paired nasopharyngeal and middle ear isolates in otitis media due to nontypable Haemophilus influenzae: pathogenetic and epidemiological observations. J Infect Dis 156, 723-731.

Murphy, T. F., Sethi, S., Klingman, K. L., Brueggemann, A. B. \& Doern, G. V. (1999). Simultaneous respiratory tract colonization by multiple strains of nontypeable Haemophilus influenzae in chronic obstructive pulmonary disease: implications for antibiotic therapy. J Infect Dis 180, 404-409.

Murphy, T. F., Brauer, A. L., Sethi, S., Kilian, M., Cai, X. \& Lesse, A. J. (2007). Haemophilus haemolyticus: a human respiratory tract commensal to be distinguished from Haemophilus influenzae. J Infect Dis $195,81-89$.

Murphy, T. F., Faden, H., Bakaletz, L. O., Kyd, J. M., Forsgren, A., Campos, J., Virji, M. \& Pelton, S. I. (2009). Nontypeable Haemophilus influenzae as a pathogen in children. Pediatr Infect Dis J 28, 43-48.
Pichichero, M. E. \& Reed, M. D. (2009). Variations in amoxicillin pharmacokinetic/pharmacodynamic parameters may explain treatment failures in acute otitis media. Paediatr Drugs 11, 243-249.

Pichichero, M. E., Doern, G. V., Kuti, J. L. \& Nicolau, D. P. (2008). Probability of achieving requisite pharmacodynamic exposure for oral beta-lactam regimens against Haemophilus influenzae in children. Paediatr Drugs 10, 391-397.

Porat, N., Arguedas, A., Spratt, B. G., Trefler, R., Brilla, E., Loaiza, C., Godoy, D., Bilek, N. \& Dagan, R. (2004). Emergence of penicillinnonsusceptible Streptococcus pneumoniae clones expressing serotypes not present in the antipneumococcal conjugate vaccine. J Infect Dis 190, 2154-2161.

Smith-Vaughan, H. C., Leach, A. J., Shelby-James, T. M., Kemp, K., Kemp, D. J. \& Mathews, J. D. (1996). Carriage of multiple ribotypes of non-encapsulated Haemophilus influenzae in aboriginal infants with otitis media. Epidemiol Infect 116, 177-183.

Spinola, S. M., Peacock, J., Denny, F. W., Smith, D. L. \& Cannon, J. G. (1986). Epidemiology of colonization by nontypable Haemophilus influenzae in children: a longitudinal study. J Infect Dis 154, 100109.

Stackebrandt, E., Frederiksen, W., Garrity, G. M., Grimont, P. A., Kämpfer, P., Maiden, M. C., Nesme, X., Rosselló-Mora, R., Swings, J. \& other authors (2002). Report of the ad hoc committee for the reevaluation of the species definition in bacteriology. Int J Syst Evol Microbiol 52, 1043-1047.

St Sauver, J., Marrs, C. F., Foxman, B., Somsel, P., Madera, R. \& Gilsdorf, J. R. (2000). Risk factors for otitis media and carriage of multiple strains of Haemophilus influenzae and Streptococcus pneumoniae. Emerg Infect Dis 6, 622-630.

Tamura, K., Dudley, J., Nei, M. \& Kumar, S. (2007). MEGA4: Molecular Evolutionary Genetics Analysis (MEGA) software version 4.0. Mol Biol Evol 24, 1596-1599.

van Schilfgaarde, M., Eijk, P., Regelink, A., van Ulsen, P., Everts, V., Dankert, J. \& van Alphen, L. (1999). Haemophilus influenzae localized in epithelial cell layers is shielded from antibiotics and antibodymediated bactericidal activity. Microb Pathog 26, 249-262.

Xu, Q., Pichichero, M. E., Casey, J. R. \& Zeng, M. (2009). Novel type of Streptococcus pneumoniae causing multidrug-resistant acute otitis media in children. Emerg Infect Dis 15, 547-551. 